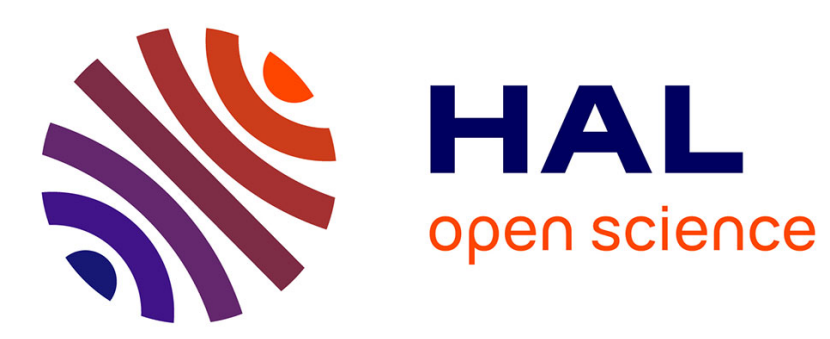

\title{
Controllable Packet Prioritization on PlanetLab Using NEPI
}

\author{
Alina Quereilhac, Claudio Freire, Thierry Turletti, Walid Dabbous
}

\section{To cite this version:}

Alina Quereilhac, Claudio Freire, Thierry Turletti, Walid Dabbous. Controllable Packet Prioritization on PlanetLab Using NEPI. 2012, 10.1007/978-3-642-35576-9_47 . hal-01280097

\section{HAL Id: hal-01280097 \\ https://hal.science/hal-01280097}

Submitted on 29 Feb 2016

HAL is a multi-disciplinary open access archive for the deposit and dissemination of scientific research documents, whether they are published or not. The documents may come from teaching and research institutions in France or abroad, or from public or private research centers.
L'archive ouverte pluridisciplinaire $\mathbf{H A L}$, est destinée au dépôt et à la diffusion de documents scientifiques de niveau recherche, publiés ou non, émanant des établissements d'enseignement et de recherche français ou étrangers, des laboratoires publics ou privés. 


\title{
Controllable packet prioritization on PlanetLab using NEPI
}

\author{
Alina Quereilhac, Claudio Freire, Thierry Turletti, and Walid Dabbous \\ INRIA, Sophia Antipolis, France. \\ \{alina.quereilhac, claudio-daniel.freire, thierry.turletti, walid.dabbous\}@ \\ inria.fr
}

\begin{abstract}
We present the extensions made to NEPI, the Network Experimentation Programming Interface, to allow easy creation and customization of routing overlays on top of PlanetLab. We particularly focus on demonstrating the traffic shaping capabilities provided by NEPI, with the use of customizable stream filters on PlanetLab overlays to induce controllable packet prioritization.
\end{abstract}

Key words: networking, overlays, PlanetLab, NEPI

\section{Demonstration}

This demonstration is intended to supplement the paper[1] accepted at TRIDENTCOM'12. We focus on demonstrating the experiment use case presented in that paper, in which we make use of stream filters as a means to control the characteristics of an overlay deployed in PlanetLab, [2] providing a realistic yet controllable environment where to test the POPI[4] tool.

NEPI [3] is an experiment management framework which provides support for design, deployment, control and gathering of results of network experiments. We added support for automating deployment and customization of routing overlays on PlanetLab, to alleviate the complexities of performing these tasks manually, and to more easily circumvent administrative limitations. NEPI automates resource discovery, node provisioning, application deployment, and creation of tunnels between the selected nodes to build the overlay network. It also provides the ability to customize traffic in the overlays by adding user defined stream filters, processing functions applied to packets traversing the overlay tunnels. They can be used to implement custom queues, packet filters or transformations, and tunnelling protocols.

To demonstrate NEPI's ability to provide a solution to existing problems when using PlanetLab, we selected a previously published experiment case [4]. In this experiment, researchers developed the POPI tool to attempt to infer packet priorities in the intervening routers between two endpoints, by inducing bulk traffic and analyzing point-to-point loss rates. However, in order to verify the results obtained after running POPI on PlanetLab, it was necessary to ask ISPs about their routing policies, because no other means were available to verify that the priorities reported by the tool corresponded to actual prioritization policies. 


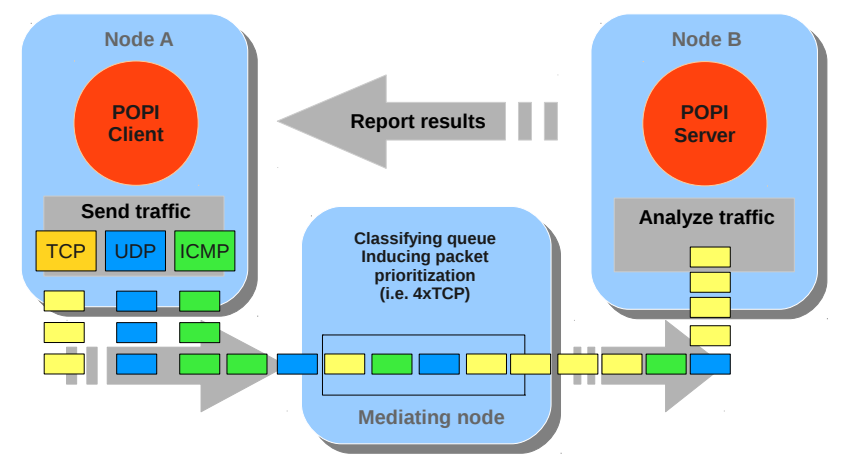

Fig. 1. POPI. An overlay is formed with 3 PlanetLab nodes. Traffic is sent by the POPI client, from node A to node B through a mediating node, where packets are processed by a classifying queue to induce class-dependent priorities. Traffic profiling results are then reported back and analyzed expecting to verify the classification.

The researcher's ability to verify the obtained results was limited, as not all providers responded, nor provided complete information.

In our demonstration we re-evaluate POPI, using NEPI to create a controlled routing overlay on PlanetLab to which we add stream filters to modify traffic behavior in a known way, by means of a classifying queue that induces packet prioritization. NEPI provides support for queues that implement queuing policies by inspecting the Type Of Service (TOS) field in the IPv4 header of the processed packets. Such queues can be easily attached to PlanetLab network devices, and thanks to this feature, designing this experiment was straightforward. Figure 1 depicts the design of our experiment consisting of 3 PlanetLab nodes connected in series. The node in the middle applies a controlled class of queuing, resulting in recognizable statistical bias to the packet stream. This method allows modification of overlay traffic behavior on PlanetLab in a controlled way, avoiding the need to request information regarding traffic policies to the ISPs.

With this experiment we demonstrate how the stream filters feature provided by NEPI enabled us to overcome existing limitations when experimenting with PlanetLab-base overlays. Moreover, apart from automating deployment of custom overlays in PlanetLab, NEPI also makes it possible to run numerous repetitions of a same experiment, or variations of it, enabling thorough experimentation.

\section{References}

1. Freire, C. et al.: Automated deployment and customization of routing overlays on PlanetLab

2. Chun, B. et al.: PlanetLab: an overlay testbed for broad-coverage services. SIGCOMM Comput. Commun. Rev. 33, 3-12 (2003)

3. Quereilhac, A. et al.: NEPI: An Integration Framework for Network Experimentation. Software, Telecommunications and Computer Networks 19, 1-5 (2011)

4. Lu, G. et al.: POPI: a user-level tool for inferring router packet forwarding priority. IEEE/ACM Trans. Netw. 18, 1-14 (2010) 